\title{
Biodiversity in the alkaloids of Turkish Papaver species*
}

\author{
Günay Sariyar ${ }^{\ddagger}$ \\ University of Istanbul, Faculty of Pharmacy, Department of Pharmacognosy, \\ 34452, Istanbul, Turkey
}

Abstract: There are about 35 Papaver species growing in Turkey, which are grouped into eight sections, namely Argemonidium, Carinata, Glauca, Miltantha, Oxytona, Papaver, Pilosa, and Rhoeadium.

As a result of our extensive work on the alkaloids of Turkish Papaver species the presence of several chemotypes has been shown. Among these chemotypes, medicinally important alkaloids such as noscapine (=narcotine), papaverine- and thebaine-containing samples have been found as new sources for the production of them.

The infraspecific variation encountered within the Papaver species has been demonstrated mainly in two sections of the genus, Miltantha and Oxytona. The alkaloids of seven species (Papaver armeniacum, $P$. curviscapum, $P$. cylindricum, $P$. fugax, $P$. persicum, $P$. polychaetum, and $P$. triniifolium) of the section Miltantha have been studied, and the existence of chemical strains has been shown mainly in P. fugax, P. persicum, and P. triniifolium.

Investigations on the species of the section Oxytona indicated that there is a relationship between the major alkaloid content and the chromosome numbers of the samples.

Isolation of new secoberbine-type alkaloids as well as promorphinane- and morphinane-types has revealed the biosynthetic relationship between the alkaloids of Miltantha and Oxytona species. Chemical races are also known for species of Papaver within other sections of the genus.

$P$. lateritium of Turkish origin from section Pilosa yielded some secoberbine and protoberberine types, whereas $P$. rhopalothece of the section Rhoeadium contained narcotine as a major alkaloid. Isolation of spirobenzylisoquinoline type from $P$. argemone (sect. Argemonidium) was the first report of the occurrence of this type in the Papaveraceae.

\section{INTRODUCTION}

Species of the genus Papaver are grouped together on the basis of their morphological characteristics into ten sections namely, Argemonidium, Carinata, Glauca, Horrida, Meconella, Miltantha, Oxytona, Papaver, Pilosa, and Rhoeadium [1-4]. With the exception of Horrida and Scapiflorae about 35 Papaver species exist in Turkey, that belong to eight sections.

Opium poppy, P. somniferum from section Papaver, has been cultivated since the Hittites in Turkey, and the production of opium, a latex obtained by incising the unripe capsules of the plant has been known for more than 2000 years. Isolation of medicinally important alkaloids, morphine, codeine, papaverine, and narcotine (=noscapine) has been of considerable interest in the alkaloids of opium.

\footnotetext{
${ }^{*}$ Lecture presented at the $3^{\text {rd }}$ IUPAC International Conference on Biodiversity (ICOB-3), Antalya, Turkey, 3-8 November 2001. Other presentations are presented in this issue, pp. 511-584.

‡E-mail:gsarıyar@superonline.com
} 
The illicit use of opium and its major alkaloid morphine as a drug of abuse led the Turkish government to ban the cultivation of P. somniferum in 1972. But increasing demand for morphine in the pharmaceutical industry was reconsidered by our government to start the cultivation again. Today, the plant is cultivated to obtain capsules for the production of morphine and its derivatives in the alkaloid factory located at Bolvadin in Central Turkey. The demand for codeine is much bigger than morphine, and this alkaloid has largely been prepared from codeine by methylization.

The increasing abuse of opiates has stimulated the search for raw materials other than P. somniferum which would meet the requirements of the pharmaceutical industry. Thus, plants containing nonaddictive thebaine as principal alkaloid could be used for the manufacture of codeine.

When the Turkish government banned the cultivation of P. somniferum in 1972, we decided to investigate the alkaloids of Papaver species growing wild in Turkey to find alternative sources for the medicinally important alkaloids as well as to evaluate the alkaloid contents of Turkish Papaver species. The location of the specimen collected in Turkey are shown in Figs. 1-4.

\section{REVIEW}

\section{Alkaloids from section Oxytona}

In Flora of Turkey, Cullen reported the existence of the following four species within the section [3].

P. bracteatum Lindl.

P. lasiothrix Fedde

P. orientale L.

P. paucifoliatum (Trautv.) Fedde

Revision of section Oxytona by Goldblatt resulted in the recognition of three species [5]. In this review, it was reported that each species could be characterized morphologically (e.g., by petal coloring and markings and by capsule shape). Furthermore, the three species were distinguished by cytological and chemical techniques:

a) $\quad$. bracteatum, $2 \mathrm{n}=14$, major alkaloid thebaine $\mathbf{7 b}$

b) $\quad$. orientale, $2 \mathrm{n}=28$, major alkaloid oripavine $7 \mathbf{a}$

c) P. pseudo-orientale, $2 \mathrm{n}=42$, major alkaloid, isothebaine $\mathbf{5 c}$

In this review, although the specimens from the locations where P. bracteatum and P. lasiothrix grow have not been obtained and the field observations have not been made, these samples have been accepted as synonyms of $P$. pseudo-orientale. P. paucifoliatum is treated as a synonym of $P$. orientale in this review.

Many specimens from this section were investigated, and the results were published [6-12]. Some confusing results have led us to extend the investigation on this section with careful field observation. Tables 1 and 2 summarize the type of the alkaloids obtained from 18 samples of $P$. pseudo-orientale and 5 samples of P.orientale. The samples of P. pseudo-orientale (P4-P16) having a chromosome number of $2 \mathrm{n}=42$ and containing aporphine $\mathbf{5}$ type, isothebaine $\mathbf{5} \mathbf{c}$ as the major alkaloid should be considered as pure species. Three samples (P1, P3, and P17) have been found to contain either promorphinane $\mathbf{6}$ type, salutaridine $\mathbf{6 d}$ and secoberbine $\mathbf{8}$ type, macrantaline $\mathbf{8 a}$ or salutaridine $\mathbf{6 d}$ and morphinane $\mathbf{7}$ type, thebaine 7a as the major alkaloids and have a chromosome number of $2 n=14$. As a result of field observations, these samples may better be treated as varieties of $P$. bracteatum having two chemical strains.

Two samples of P. pseudo-orientale from Northeastern Turkey (P2 and P18) contain either salutaridine $6 \mathbf{d}$ and mecambridine $9 \mathbf{d}$ (protoberberine 9 type) or salutaridine $\mathbf{6 d}$ and papaveroxine $\mathbf{8 e}$ (secoberberine 8 type). The chromosome number of $\mathrm{P} 2$ was determined as $2 \mathrm{n}=28$. This sample has spreading hairs on the peduncle and was previously identified as $P$. lasiothrix by Cullen. The field observations suggest that both samples could be intermediates between P. bracteatum and P. pseudo-orientale. 
Table 1 Major alkaloids from Papaver species of the section Oxytona in Turkey.

\begin{tabular}{|c|c|c|c|c|}
\hline Species & & Major alkaloid & $\begin{array}{c}\text { Diploid } \\
\text { chromosome } \\
\text { number }(2 n)\end{array}$ & Locality \\
\hline \multirow{2}{*}{$\begin{array}{l}\text { P. bracteatum* } \\
\text { " }\end{array}$} & P3 & salutaridine, thebaine & 14 & B7 TUNCELİ \\
\hline & $\mathrm{P} 1$ & salutaridine, thebaine & 14 & B5 NİĞDE \\
\hline P. lasiothrix** & $\mathrm{P} 2$ & mecambridine, salutaridine & 28 & A7 GÜMÜŞHANE \\
\hline \multirow[t]{5}{*}{ P. orientale } & 01 & oripavine & & A9 KARS \\
\hline & 02 & oripavine & & A9 KARS \\
\hline & 03 & mecambridine & & A8 ERZURUM \\
\hline & 04 & oripavine & 28 & B9 AĞRI \\
\hline & 05 & oripavine & & A9 KARS \\
\hline P. pseudo-orientale & P4 & $\begin{array}{l}\text { isothebaine, mecambridine, } \\
\text { orientalidine }\end{array}$ & & A7 GÜMÜŞHANE \\
\hline$"$ & P5 & $\begin{array}{l}\text { isothebaine, mecambridine, } \\
\text { orientalidine }\end{array}$ & & A8 ERZURUM \\
\hline$"$ & P6 & $\begin{array}{l}\text { isothebaine, mecambridine, } \\
\text { orientalidine }\end{array}$ & & A9 KARS \\
\hline$"$ & P7 & $\begin{array}{l}\text { isothebaine, mecambridine, } \\
\text { orientalidine }\end{array}$ & & A9 KARS \\
\hline$"$ & P8 & $\begin{array}{l}\text { isothebaine, mecambridine, } \\
\text { orientalidine }\end{array}$ & & A9 KARS \\
\hline$"$ & P9 & $\begin{array}{l}\text { isothebaine, mecambridine, } \\
\text { orientalidine }\end{array}$ & & A9 KARS \\
\hline$"$ & P10 & isothebaine, mecambridine & 42 & A9 KARS \\
\hline$"$ & P11 & isothebaine, mecambridine & & B9 AĞRI \\
\hline$"$ & $\mathrm{P} 12$ & isothebaine, mecambridine & & A9 AĞRI \\
\hline$"$ & P13 & isothebaine, mecambridine & 42 & A9 KARS \\
\hline$"$ & $\mathrm{P} 14$ & isothebaine, mecambridine & 42 & B9 VAN \\
\hline$"$ & P15 & isothebaine, mecambridine & & B9 VAN \\
\hline$"$ & $\mathrm{P} 16$ & isothebaine & 42 & C10 HAKKARİ \\
\hline$"$ & $\mathrm{P} 17^{* *}$ & $\begin{array}{l}\text { salutaridine, macranthaline } * * *, \\
\text { macrantaldehyde } * * *\end{array}$ & 14 & B7 SIVAS \\
\hline$"$ & P18 & salutaridine, papaveroxine $* * *$ & & A7 GİRESUN \\
\hline
\end{tabular}

* P. pseudo-orientale, Goldblatt 1974.

**This species may better be treated as a variety of $P$. bracteatum or of $P$. orientale.

*** Novel alkaloids

Table 2 Types of alkaloids in the section Oxytona.

\begin{tabular}{|c|c|c|c|c|}
\hline & P. bracteatum & P. lasiothrix & P. orientale & P. pseudo-orientale \\
\hline \multicolumn{5}{|c|}{ Simple isoquinoline 1} \\
\hline Cotarnine 1a & & & & + \\
\hline Cotarnoline $\mathbf{1 b}$ & & & & + \\
\hline \multicolumn{5}{|l|}{ Proaporphine 4} \\
\hline Orientalinone & & & & + \\
\hline \multicolumn{5}{|l|}{ Aporphine 5} \\
\hline Bracteoline & & & & + \\
\hline Isothebaine $\mathbf{5 c}$ & + & & + & + \\
\hline \multicolumn{5}{|l|}{ Promorphinane 6} \\
\hline Salutadimerine* $\mathbf{6 e}$ & & + & & + \\
\hline
\end{tabular}


Table 2 (Continued)

\begin{tabular}{|c|c|c|c|c|}
\hline & P. bracteatum & P. lasiothrix & P. orientale & P. pseudo-orientale \\
\hline Salutaridine 6d & + & + & + & + \\
\hline Salutaridine- $N$-oxide* & + & + & & \\
\hline Norsalutaridine & & + & & \\
\hline \multicolumn{5}{|l|}{ Morphinane 7} \\
\hline Oripavine $\mathbf{7 a}$ & + & & + & \\
\hline Neopine & + & & & \\
\hline Thebaine $\mathbf{7 b}$ & + & + & + & \\
\hline Thebaine-N-oxide & + & + & & \\
\hline \multicolumn{5}{|l|}{ Secoberbine 8} \\
\hline Macrantaline $*$ 8a & & & & + \\
\hline Macrantaldehyde* $8 \mathbf{b}$ & & & & + \\
\hline Macrantoridine* 8c & & & & + \\
\hline Narcotindiol* 8g & & & & + \\
\hline Papaveroxine* 8e & & & & + \\
\hline Papaveroxinoline* 8d & & & & + \\
\hline Papaveroxidine* $\mathbf{8 f}$ & & & & + \\
\hline \multicolumn{5}{|l|}{ Protoberberine 9} \\
\hline Mecambridine 9d & & + & + & + \\
\hline Orientalidine $9 \mathbf{e}$ & & + & + & + \\
\hline \multicolumn{5}{|l|}{$N$-methopapaverberbine* 9c } \\
\hline \multicolumn{5}{|l|}{ Phthalideisoquinoline 11} \\
\hline Narcotine 11b & & & & + \\
\hline Narcotinhemiacetale* 11a & & & & + \\
\hline Narcotolinol* 11c & & & & + \\
\hline \multicolumn{5}{|l|}{ Rhoeadine 13} \\
\hline Alpinigenine & & & + & + \\
\hline Alpinine & & & & + \\
\hline
\end{tabular}

*Novel alkaloids

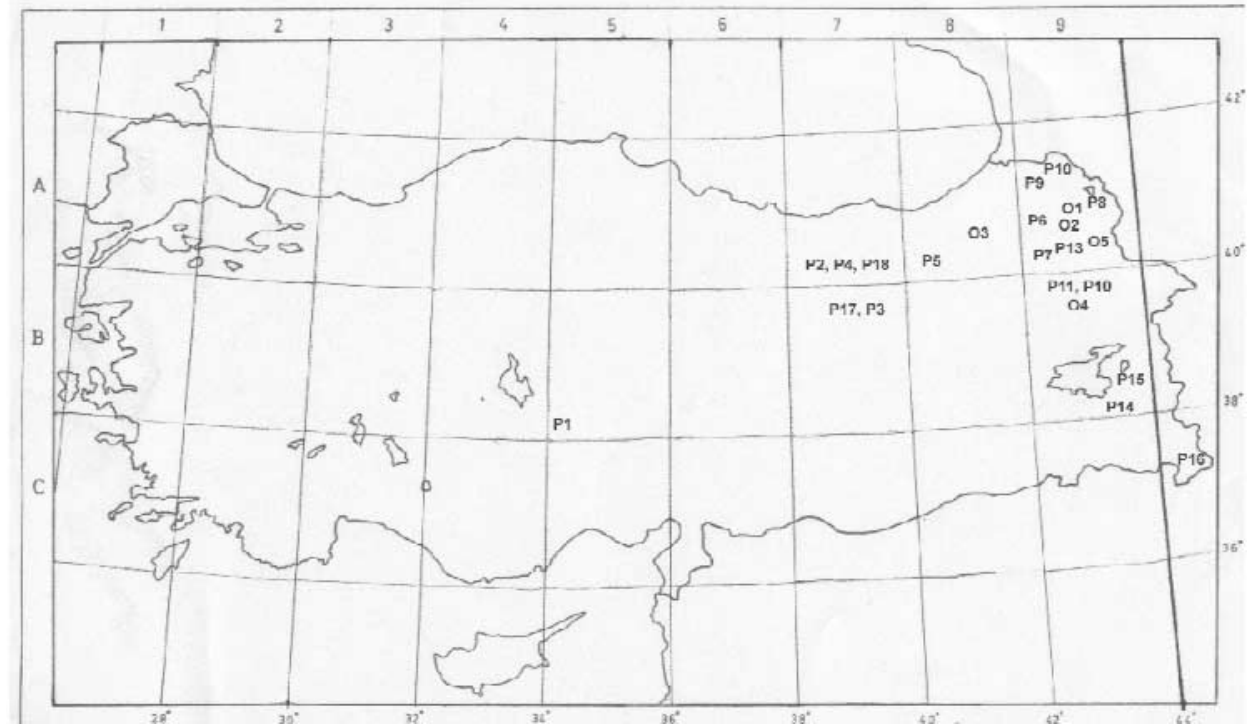

Fig. 1 Distribution of Papaver pseudo-orientale (P1-P18) and Papaver orientale (O1-O5). 
Four samples of $P$. orientale (01, 02, 04, and 05) yielded oripavine 7a, whereas one sample (03) was found to contain mecambridine $9 \mathbf{d}$ as the major alkaloid. The chromosome number of 01 and 02 was determined as $2 n=28$. Although the chromosome number of 03 has not been reported, there was no difference between 03 and other samples of $P$. orientale morphologically. Hence, we accept 03 as a chemical strain of $P$. orientale.

Alkaloids of $P$. paucifoliatum of Turkish origin accepted as a synonym of $P$. orientale by Goldblatt have not been investigated yet.

\section{Alkaloids from section Miltantha}

According to Cullen, the following species exist in Turkey [3].

P. acrochaetum Bornm.

P. $\operatorname{armeniacum}($ L.) DC.

P. curviscapum Nabelek

P. cylindricum Cullen

P. fugax Poir.

P. persicum Lindl. (syn.: P. tauricola. Boiss.)

P. polychaetum Schott \& Kotschy

P. triniifolium Boiss.

With the exception of $P$. acrochaetum, the alkaloids of seven species have extensively been investigated $[10,12-20]$. Tables 3 and 4 summarize the alkaloid content of the species with their types.

Two samples of P. armeniacum ( $\mathrm{Al}$ and A2) were investigated and found to contain either rhoeadine $\mathbf{1 3}$ or proaporphine $\mathbf{4}$-aporphine $\mathbf{5}$-benzylisoquinoline $\mathbf{2}$ types as the major alkaloid that indicated the presence of two chemotypes.

Table 3 Major alkaloids from Papaver species of the section Miltantha in Turkey.

\begin{tabular}{|c|c|c|c|}
\hline Species & & Major alkaloid & Locality \\
\hline P. armeniacum & A1 & oreodine, rhoeadine & C9 HAKKARİ \\
\hline$"$ & $\mathrm{~A} 2$ & floripavidine, mecambrine, papaverine & B9 VAN \\
\hline P. cylindricum & & $\begin{array}{l}\text { narcotine, oripavine, } \\
\text { rhoeadine, thebaine }\end{array}$ & B9 VAN \\
\hline$"$ & & narcotine & B9 AĞRI \\
\hline P. curviscapum & & berberine, protopine & C9 HAKKARİ \\
\hline P. fugax & $\mathrm{F} 1$ & theabine, narcotine & B8 BİNGÖL \\
\hline$"$ & $\mathrm{~F} 2$ & floripavidine, mecambrine & A8 GÜMÜŞHANE \\
\hline$"$ & $\mathrm{~F} 3$ & armepavine, oreodine, rhoeadine & A9 KARS \\
\hline$"$ & $\mathrm{~F} 4$ & floripavidine, salutaridine & A9 KARS \\
\hline " & F5 & $\begin{array}{l}\text { narcotine, narcotinehemiacetale, } \\
\text { papaveroxine }\end{array}$ & C9 HAKKARİ \\
\hline$"$ & F6 & oreodine, rhoeadine & C9 HAKKARİ \\
\hline P. persicum* & $\mathrm{P} 1$ & oreodine, oreogenine & B7 MALATYA \\
\hline$"$ & $\mathrm{P} 2$ & glaucamine, glaudine & B5 KAYSERİ \\
\hline$"$ & P3 & rhoeagenine & C5 ADANA \\
\hline$"$ & $\mathrm{P} 4$ & mecambridine, salutaridine & B7 ERZINCAN \\
\hline$"$ & P5 & oreodine, rhoeadine & B7 SIVAS \\
\hline$"$ & P6 & salutaridine, floripavidine & A8 GÜMÜŞHANE \\
\hline$"$ & P7 & armepavine, floripavidine & C9 HAKKARİ \\
\hline P. polychaetum & Po & berberine & MERSİN \\
\hline
\end{tabular}

(continues on next page) 
Table 3 (Continued)

\begin{tabular}{clll}
\hline Species & & \multicolumn{1}{c}{ Major alkaloid } & \multicolumn{1}{c}{ Locality } \\
\hline P. triniifolium & T1 & floripavidine, rhoeadine & B5 NiĞGE \\
$"$ & T2 & $\begin{array}{l}\text { oreodine, rhoeadine, thebaine } \\
\text { mecambrine, narcotine, papaverine }\end{array}$ & B8 ERZURUM \\
$"$ & T3 & B7 ERZINCAN \\
$"$ & T4 & oreodine, thebaine & B7 ERZINCAN \\
$"$ & T5 & mecambrine, oreodine, rhoeadine & B7 TUNCELI \\
$"$ & T6 & oreodine,rhoeadine & B7 TUNCELI \\
$"$ & T7 & oreodine & B7 MALATYA \\
& T8 & oreogenine, rhoeagenine & B4 ANKARA \\
\hline
\end{tabular}

*This species is previously treated as P. tauricola

Table 4 Types of alkaloids in the section Miltantha.

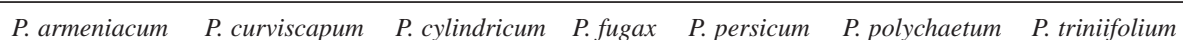

Benzylisoquinoline 2

Armepavine 2a

Crykonisine

Miltanthaline* $2 \mathbf{b}$

Papaverine 2c

Isopavine $\mathbf{3}$

Amurensinine 3a

Proaporphine 4

Mecambrine 4a

$N$-methylcrotonosine

Aporphine 5

Floripavidine $\mathbf{5 b}$

$\mathrm{N}$-methylasimilobine

Promorphinane 6

Amurine

Salutaridine 6d

Morphinane 7

Oripavine 7a

Thebaine 7b

$\mathrm{N}$-methylthebaine

Secoberbine 8

Papaveroxine 8e

Protoberberine (with

Tetrahydroproto-

berberine) 9

Berberine 9a

Cheilantifoline

Isocorypalmine

$N$-methylsinactine

Sinanctine

Scoulerine

Protopine 10

Allocryptopine 10b

1-Methoxy-

allocryptopine* 10c

1-Methoxy-13-oxo-

allocryptopine

Protopine 10a

Phthalideisoquinoline 11 
Table 4 (Continued)

\begin{tabular}{|c|c|c|c|c|c|c|c|}
\hline & P. armeniacum & P. curviscapum & P. cylindricum & P. fugax & P. persicum & P. polychaetum & P. triniifolium \\
\hline Narceine & & & & & & & + \\
\hline Narcotine 11b & + & & + & + & & & + \\
\hline Narcotinhemiacetale* 11a & & & & + & & & \\
\hline$N$-Methylnarcotine* 11d & & & + & & & & \\
\hline \multicolumn{8}{|l|}{ Rhoeadine 13} \\
\hline Epiglaudine & & & & & + & & \\
\hline Glaucamine 13e & & & & & + & & \\
\hline Glaudine & & & & & + & & \\
\hline Oreodine 13b & + & & & + & + & & + \\
\hline Oreogenine 13d & & & & & + & & + \\
\hline$O$-Ethylrhoeagenine & & & & & & & + \\
\hline$O$-Ethyloreogenine & & & & & & & + \\
\hline$O$-Ethyltriniifoline & & & & & & & + \\
\hline Rhoeadine 13a & + & & + & + & + & & + \\
\hline Rhoeagenine 13c & & & & & + & & + \\
\hline Triniifoline* 13f & & & & & & & + \\
\hline
\end{tabular}

*Novel alkaloids

The samples of P. fugax (F1-F6) and P. persicum (P1-P7) have been found to contain either proaporphine 4-aporphine $\mathbf{5}$, proaporhine 4 -aporphine 5 - benzylisoquinoline $\mathbf{2}$, aporphine $\mathbf{5}$, benzyltetrahydroisoquinoline $\mathbf{2}$-aporphine $\mathbf{5}$, proaporphine 4-promorphinane $\mathbf{6}$, aporphine 5-promorphinane $\mathbf{6}$, secoberbine 8 phthalideisoquinoline 11, benzyl-tetrahydroisoquinoline 2-rhoeadine 13, or rhoeadine 13 types as the major alkaloids. As a result, $P$. fugax and P. persicum of Turkish origin have been shown to exist in six and four different chemical strains, respectively.

The alkaloids of $P$. triniifolium, an endemic species of the section have been isolated from eight samples (T1-T8). Five different chemical strains have been found in this species containing either aporphine 5-rhoeadine 13, aporphine 5-phthalideisoquinoline 14-benzlisoquinoline 2, proaporphine 4-rhoeadine 13, rhoeadine 13-morphinane 7, or rhoeadine 13 types.

Distribution of this species extends from Central to East Turkey (Fig. 2).

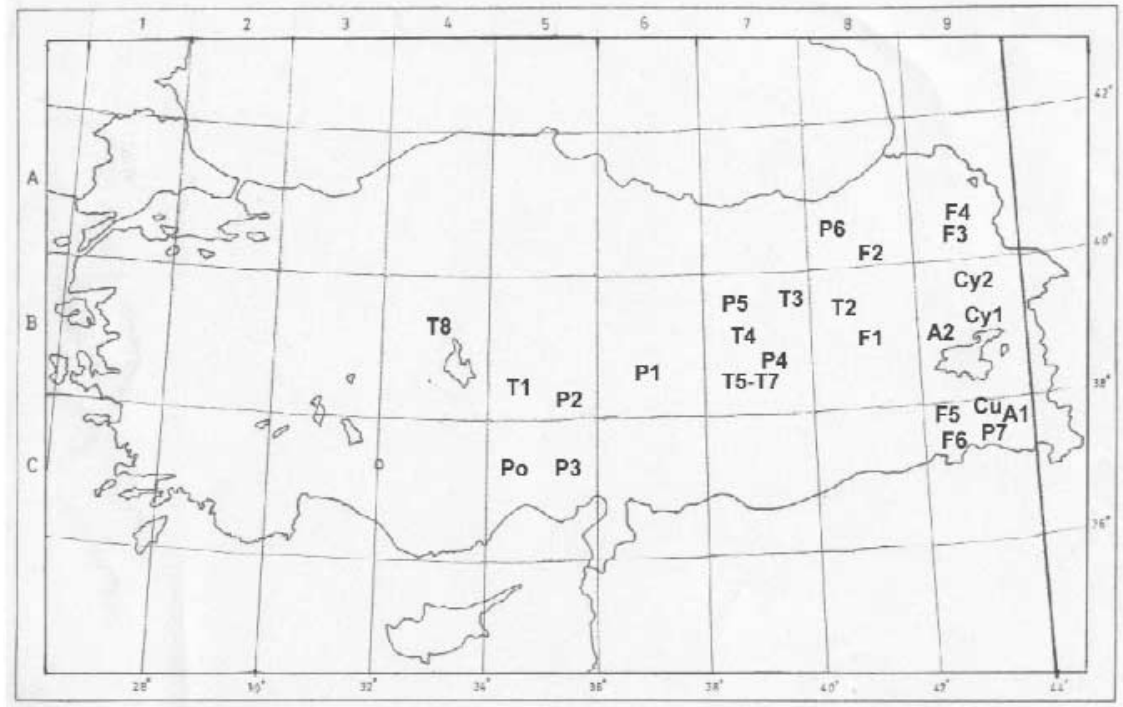

Fig. 2 Distribution of P. armeniacum (A1-A2), P. curviscapum (Cu), P. cylindricum (Cy), P. fugax (F1-F6), P. persicum (P1-P7), P. polychaetum (Po), P. triniifolium (T1-T8). 
Two samples of $P$. cylindricum contained morphinane $\mathbf{7}$ (oripavine 7a) together with phthalideisoquinoline 11 (narcotine 11b) and rhoeadine 13 (rhoeadine 13a) types.

The remaining two species of the section Miltantha, P. polychaetum and P. curviscapum, both yielded berberine 9a as the major alkaloid. They also have morphologically similarities being subscapose plants bearing solitary flowers on long peduncles.

The existence of secoberbine $\mathbf{8}$ type (papaveroxine $\mathbf{8 e}$ ) has been reported from Miltantha section for the first time.

The presence of promorphinane $\mathbf{6}$, morphinane 7 , phthalideisoquinoline 11, and secoberbine 8 types as the major alkaloid in section Miltantha indicates the close relationship between Oxytona and Miltantha.

Chromosome numbers of some species have been determined, however, there was no relationship between the chromosome number and the major alkaloid content in section Miltantha.

The results of our recent investigations on sections Miltantha and Oxytona are the contributions to the review article published by J. D. Phillipson, who indicated the infraspecific variations in two sections of the genus Papaver [20].

\section{Alkaloids from section Pilosa}

Section Pilosa contains five species that are endemic to Turkey [3].

P. apokrinomenon Fedde

P. lateritium Koch

P. pilosum Sibth. \& Sm.

P. spicatum Boiss. \& Bal.

P. strictum Boiss. \& Bal.

Of these species, P. lateritium is in a different subgroup than the other four species having different morphological characters and different type of major alkaloids. The alkaloids of this section were investigated in detail [21-23] and the results have been summarized in Tables 5 and 6 . The species in this section have no chemotypes. The new alkaloids of promorphinane $\mathbf{6}$ type (amurinine $\mathbf{6} \mathbf{b}$ and epiamurinine $\mathbf{6 c}$ ) alkaloids have been found in this section.

Table 5 Major alkaloids from Papaver species of the section Pilosa in Turkey.

\begin{tabular}{llcl}
\hline Species & \multicolumn{1}{c}{ Major alkaloid } & $\begin{array}{c}\text { Diploid } \\
\text { chromosome } \\
\text { number (2n) }\end{array}$ & Locality \\
\hline P. apokrinomenon & amurine, roemerine, n-methyllaurotetanine & & B4 KONYA \\
$P$. lateritium & protopine, rhoeadine, rhoeagenine & 14 & A8 RIZE \\
$P$. pilosum & amurine, glaucine, roemerine & 28 & A2 BURSA \\
$P$ spicatum var. luschanii & glaucine, roemerine & & C3 ANTALYA \\
var. spicatum & glaucine, roemerine & & C3 ANTALYA \\
$P$. strictum & amurine & 14 & B2 KÜTAHYA
\end{tabular}

Table 6 Types of alkaloids in the section Pilosa.

\begin{tabular}{|c|c|c|c|c|c|}
\hline & P. apokrinomenon & P. lateritium & P. pilosum & P. spicatum & P. strictum \\
\hline \multicolumn{6}{|l|}{ Aporphine 5} \\
\hline Dehydroglaucine & + & & + & + & + \\
\hline Dehydroroemerine & + & & + & + & + \\
\hline
\end{tabular}


Table 6 (Continued)

\begin{tabular}{|c|c|c|c|c|c|}
\hline & P. apokrinomenon & P. lateritium & P. pilosum & P. spicatum & P. strictum \\
\hline Glaucine $\mathbf{5 f}$ & + & & + & + & + \\
\hline$N$-methylglaucine & & & & & + \\
\hline$N$-methyllaurotetanin $\mathbf{5 g}$ & + & & & & + \\
\hline$N$-methylroemerine & & & & & + \\
\hline Roemerine $\mathbf{5 a}$ & + & & + & + & + \\
\hline \multicolumn{6}{|l|}{ Promorphinane 6} \\
\hline Amurine $\mathbf{6 a}$ & & & + & + & + \\
\hline Amurinine* $\mathbf{6 b}$ & & & + & + & + \\
\hline Dihydronudaurine & & & + & + & + \\
\hline Epiamurinine* $\mathbf{6 c}$ & & & + & + & + \\
\hline \multicolumn{6}{|l|}{ Secoberbine 8} \\
\hline Macranthaline 8a & & + & & & \\
\hline \multicolumn{6}{|l|}{ Protoberberine 9} \\
\hline Mecambridine 9d & & + & & & \\
\hline$N$-Methyltetrahydropalmatine & & + & & & \\
\hline \multicolumn{6}{|l|}{ Protopine 10} \\
\hline Protopine 10a & & + & & & \\
\hline Cryptopine & & + & & & \\
\hline \multicolumn{6}{|l|}{ Rhoeadine 13} \\
\hline Rhoeadine 13a & & + & & & \\
\hline Rhoeagenine & & + & & & \\
\hline
\end{tabular}

*New alkaloids

The existence of secoberbine $\mathbf{8}$ alkaloid macranthaline $\mathbf{8 a}$ has been shown in this section for the first time.

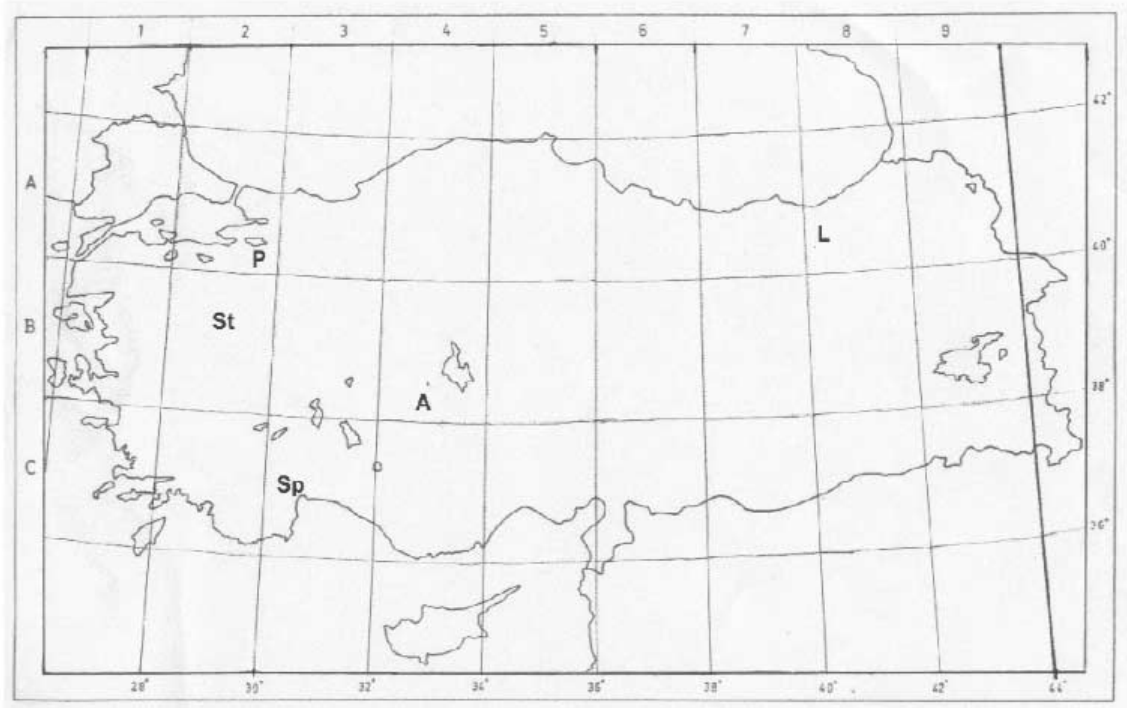

Fig. 3 Distribution of P. apokrinomenon (A), P. lateritium (L), P. pilosum (P), P. spicatum (Sp), and P. strictum (St). 


\section{Alkaloids from section Rhoeadium}

According to Cullen and Kadereit, the following species exist in Turkey [3,24]:

Cullen 1965

P. arenarium Bieb.

P. clavatum Boiss. \& Hausskn. ex Boiss.

P. commutatum Fisher \& Meyer

P. dubium L.

P. lacerum Popov

P. postii Fedde

P. rhoeas L.

P. rhopalothece Stapf.

P. stylatum Boiss. \& Bal.

P. syriacum Boiss.
Kadereit 1988

P. purpureamarginatum Kadereit

P. dubium L. ssp. dubium ssp. laevigatum (syn.: P. lacerum) ssp. lecoquii

P. arachnoideum Kadereit

P. arenarium M. Bieb

P. commutatum Fisher \& C. Meyer ssp. euxinum Kadereit

P. guerlekense Stapf. (syn.: P. rhopalothece)

P. stylatum Boiss. \& Bal.

P. clavatum Boiss. \& Hausskn. ex Boiss.

P. rhoeas L.

The alkaloids isolated from this section have been summarized in Table 7. Two major alkaloids aporphine 5 (isocorydine 5c) and phthalideisoquinoline 11 (narcotine 11b), types have been isolated from P. guerlekense (syn.: P. rhopalothece). The presence of narcotine as the major alkaloid in this species explains the traditional use of the plant as antitussive [25].

Table 7 Major alkaloids from Papaver species of the section Rhoeadium.

\begin{tabular}{|c|c|c|c|}
\hline Species & & Major alkaloid & Locality \\
\hline \multirow[t]{2}{*}{ P. commutatum ssp. euxinum } & $\mathrm{C} 1^{*}$ & isocorydine & A6 ORDU \\
\hline & $\mathrm{C} 2^{*}$ & isocorydine & A4 ZONGULDAK \\
\hline P. dubium ssp. dubium & & berberine, thalifendine & A3 BOLU \\
\hline ssp. laevigatum & & berberine & A3 BOLU \\
\hline ssp. lecoqii & & allocrytopine, protopine & C1 MUĞLA \\
\hline P. lacerum ${ }^{* *}$ & & roemerine & B5 KAYSERİ \\
\hline P. rhoeas & & rhoeadine & A6 ORDU \\
\hline P. rhopalothece $e^{* * *}$ & & isocorydine, narcotine & C2 MUĞLA \\
\hline
\end{tabular}

\footnotetext{
* Different minor alkaloids

${ }^{* * *}$ This species is treated as a subspecies, P. dubium ssp. laevigatum (Kadareit 1988)

${ }^{* * * *}$ Synonym of $P$. guerlekense
}

Table 8 Types of alkaloids isolated from Turkish Papaver species, section Rhoeadium.

\begin{tabular}{|c|c|c|c|c|c|c|}
\hline & $\begin{array}{l}\text { P. commutatum } \\
\text { ssp. } \\
\text { commutatum }\end{array}$ & $\begin{array}{c}\text { P. dubium } \\
\text { ssp. dubium }\end{array}$ & $\begin{array}{c}\text { P. dubium } \\
\text { ssp. laevigatum }\end{array}$ & P. lacerum & P. rhoeas & P. rhopalothece \\
\hline \multicolumn{7}{|l|}{ Isopavine 3} \\
\hline Amurensinine $\mathbf{3 a}$ & + & & & & & \\
\hline \multicolumn{7}{|l|}{ Proaporphine 4} \\
\hline Mecambrine $\mathbf{4 a}$ & & & + & + & & \\
\hline Pronuciferine & & & & + & & \\
\hline \multicolumn{7}{|l|}{ Aporphine 5} \\
\hline Corydine & & + & & & & \\
\hline Isocorydine $\mathbf{5 d}$ & + & + & & & + & \\
\hline
\end{tabular}


Table 8 (Continued)

\begin{tabular}{|c|c|c|c|c|c|c|}
\hline & $\begin{array}{l}\text { P. commutatum } \\
\text { ssp. } \\
\text { commutatum }\end{array}$ & $\begin{array}{c}\text { P. dubium } \\
\text { ssp. dubium }\end{array}$ & $\begin{array}{c}\text { P. dubium } \\
\text { ssp. laevigatum }\end{array}$ & P. lacerum & P. rhoeas & P. rhopalothece \\
\hline$N$-Methylasimilobine & & & & + & & \\
\hline Roemerine $\mathbf{5 a}$ & & + & + & & + & + \\
\hline Rhopalotine* 5e & & & & & & + \\
\hline \multicolumn{7}{|l|}{$\begin{array}{l}\text { Protoberberine } 9 \\
\text { (with tetrahydro- } \\
\text { protoberberine) }\end{array}$} \\
\hline Berberine 9a & & + & & & + & + \\
\hline Cheilantifoline & + & & & & + & \\
\hline Coptisine & & & & & & + \\
\hline Sinactine & & & & & + & \\
\hline Stylopine & & + & & & & \\
\hline $\begin{array}{l}\text { Tetrahydro } \\
\text { pseudocoptisine }\end{array}$ & & + & & & & \\
\hline Thalifendine 9b & & + & & & & \\
\hline \multicolumn{7}{|l|}{ Protopine 10} \\
\hline Allocryptopine $\mathbf{1 0 b}$ & & & + & & + & \\
\hline Couteropine & & & & & & + \\
\hline Cryptopine & & & & & & + \\
\hline Protopine 10a & & & & & + & + \\
\hline \multicolumn{7}{|l|}{$\begin{array}{l}\text { Phthalideisoquino- } \\
\text { line } 11\end{array}$} \\
\hline Narceine & & & & & & + \\
\hline Narcotine 11b & & & & & & + \\
\hline \multicolumn{7}{|l|}{$\begin{array}{l}\text { Rhoeadine and } \\
\text { Papaverrubine } 13\end{array}$} \\
\hline Papaverrubine A & + & & & & & \\
\hline Rhoeagenine 13c & + & & & & & \\
\hline Rhoeadine 13a & & & & + & & \\
\hline
\end{tabular}

* Novel alkaloids

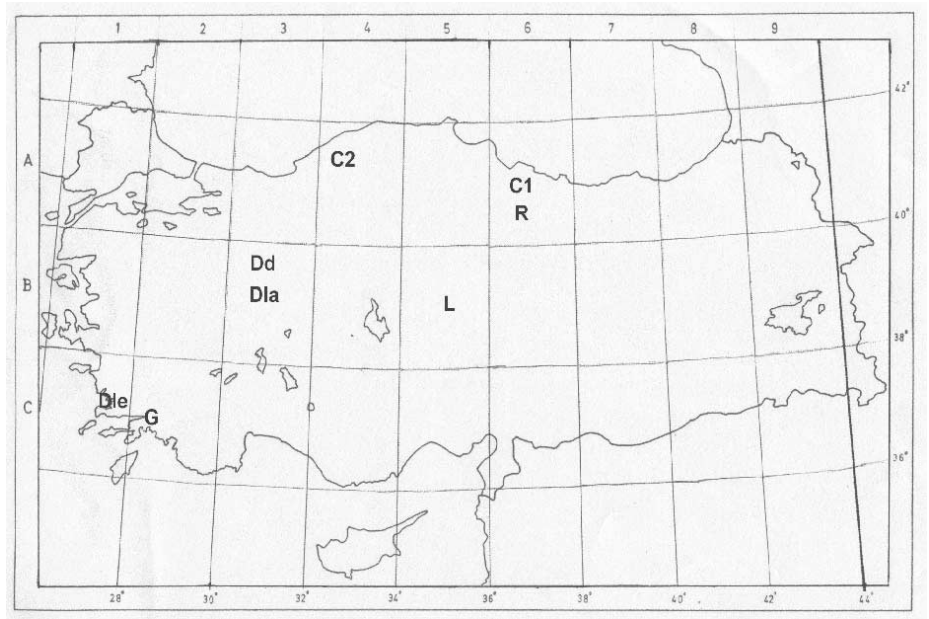

Fig. 4 Distribution of $P$. commutatum ssp. euxinum (C1-C2), P. dubium subsp. dubium (Dd), -ssp. laevigatum (Dla), -ssp. lecoqii (Dle), P. lacerum (L), P. rhoeas (R), P. rhopalothece (syn. P. guerlekense) (G).

(C) 2002 IUPAC, Pure and Applied Chemistry 74, 557-574 
Three subspecies of $P$. dubium, ssp. dubium, ssp. Laevigatum, and ssp. lecoqii have been found to contain different major alkaloids [26].

\section{Alkaloids from sections Argemonidium and Carinatae}

Cullen and Kadereit have reported the existence of the following species in the section Argemonidium in Turkey $[3,28]$. Section Carinatae is represented by only one species named as P. macrostomum Boiss. \& Huet ex Boiss. The alkaloids isolated from sections Argemonidium and Carinatae are shown in Table 9.

Cullen 1965

$P$. argemone L.

P. hybridum L.

P. virchowii Aschers \& Sin. ex Boiss.

\author{
Kadereit 1986 \\ P. argemone L. ssp. davisii Kadereit \\ ssp. minus (Boiv.) Kadereit \\ ssp. nigrotinctum (Fedde) Kadereit
}

P. hybridum L.

Table 9 Alkaloids from Papaver species of the sections Argemonidium and Carinatae.

\begin{tabular}{llc}
\hline Species & \multicolumn{1}{c}{ Major alkaloid } & \multicolumn{1}{c}{ Locality } \\
\hline P. argemone & protopine, fumariline, fumarophycine & A1 ÇANAKKALE \\
$P$. macrostomum & isocorydine, amurensine & A4 ZONGULDAK
\end{tabular}

Fumariline 12a and fumarophycine 12b (spirobenzylisoquinoline $\mathbf{1 2}$ type) isolated from P. argemone have been found for the first time in the Papaveraceae.

As a result of our investigations on Turkish Papaver species, the infraspecific variation has been demonstrated in Turkish sections Oxytona, Miltantha, and Rhoeadium. However, further investigations are necessary on some sections.

\section{REFERENCES}

1. F. Fedde. Das Pflanzenreich, A. Engler (Ed.), Part IV, No. 40, p. 288, Engelman, Leipzig (1910).

2. K. F. Gunther. Flora (Jena) 164, 393 (1975).

3. J. Cullen. In Flora of Turkey and the East Aegean Islands, P. H. Davis (Ed.), I, p. 213, University Press, Edinburgh (1995).

4. P. H. Davis, R. R. Mill, K. Tan. Flora of Turkey and the East Aegean Islands 10, p. 23 (1988).

5. P. Goldblatt. Annals of the Missouri Botanic Garden 61, 265 (1974).

6. G. Sariyar. J. Fac. Pharm. Istanbul 13, 171 (1977).

7. G. Sariyar and J. D. Phillipson. Phytochemistry 16, 2009 (1977).

8. G. Sariyar and T. Baytop. Planta Med. 38, 378 (1980).

9. J. D. Phillipson, A. Scutt, A. Baytop, N. Özhatay, G. Sariyar. Planta Med. 43, 261 (1981).

10. G. Sariyar and M. Shamma. Phytochemistry 25, 2403 (1986).

11. G. Sariyar and M. Shamma. J. Nat. Prod. 51, 802 (1988).

12. G. Sariyar, A. Freyer, H. Guinaudeau, M. Shamma. J. Nat. Prod. 53, 1302 (1990).

13. J. D. Phillipson, G. Sariyar, T. Baytop. Phytochemistry 12, 2431 (1973).

14. G. Sariyar and J. D. Phillipson. Phytochemistry 19, 2189 (1980).

15. G. Sariyar. Doğa Bilim Dergisi: Temel Bilim 7, 93 (1983).

16. G. Sariyar. Planta Med. 46, 1975 (1982).

17. G. Sariyar. Planta Med. 49, 43 (1983).

18. J. D. Phillipson, O. O. Thomas, A. I. Gray, G. Sariyar. Planta Med. 41, 105 (1981).

19. G. Sarıyar, T. Baytop, J. D. Phillipson. Planta Med. 55, 89 (1989). 
20. J. D. Phillipson. Planta Med. 48, 1687 (1983).

21. G. Sariyar and A. Öztekin. Plantes Med. Phytothér. 15, 160 (1980).

22. R. Hocquemiller, A. Öztekin, F. Roblot, M. Hutin, A. Cavé. J. Nat. Prod. 47, 342 (1984).

23. A. Öztekin, A. Baytop, M. Hutin, J. P. Foucher, R. Hocquemiller, A. Cavé. Planta Med., 51, 431 (1985).

24. J. W. A. Kadereit. Notes RBG Edinburgh 45, 225 (1988).

25. G. Sariyar and Y. N. Kalav. Planta Med. 56, 232 (1990).

26. A. Mat, G. Sariyar, Ç. Ünsal, A. Deliorman, M. Atay, N. Özhatay. Nat. Prod. Lett. 14, 205 (2000).

27. A. Sari and G. Sariyar. Planta Med. 63, 575 (1997).

28. J. W. A. Kadereit. Notes RBG, Edinburgh 44 (1), 29 (1986). 


\section{SIMPLE ISOQUINOLINE 1}<smiles>COc1c2c(cc3c1OCO3)CC[N+](C)=C2</smiles>

1a<smiles>CN1C=C2C(=O)C3=C(C=C2CC1)OCO3</smiles>

$1 \mathrm{~b}$

BENZYLISOQUINOLINE 2<smiles>COc1cc2c(cc1OC)C(Cc1ccc(O)cc1)N(C)CC2</smiles>

2a<smiles>COc1ccc(CC2c3c(cc(OC)c(OC)c3O)CCN2C)cc1</smiles>

2b<smiles>COc1ccc(Cc2nccc3cc(OC)c(OC)cc23)cc1OC</smiles>

ISOPAVINE 3

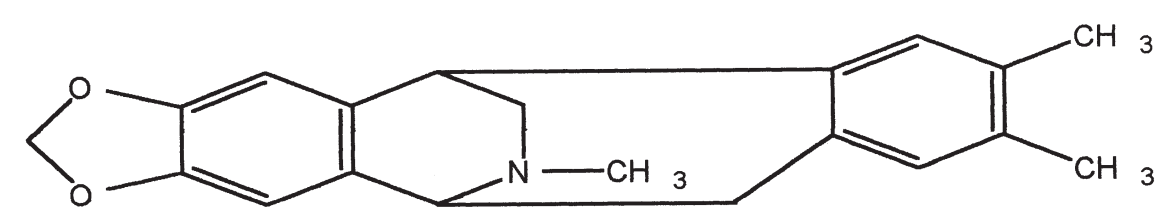

$3 \mathbf{a}$

(C) 2002 IUPAC, Pure and Applied Chemistry 74, 557-574 
PROPAPORPHINE 4

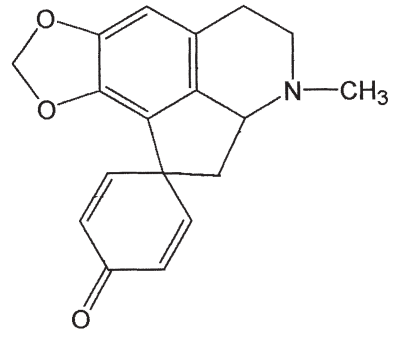

$4 a$

APORPHINE 5<smiles>[R6]c1cc2c(c([R])c1[R5])-c1c(cc([R2])c([R])c1[R3])N(C)CC2[R1]</smiles>

\begin{tabular}{ccccccc} 
& $\mathrm{R}_{1}$ & $\mathrm{R}_{2}$ & $\mathrm{R}_{3}$ & $\mathrm{R}_{4}$ & $\mathrm{R}_{5}$ & $\mathrm{R}_{6}$ \\
$\mathbf{5 a}$ & $\mathrm{H}$ & \multicolumn{2}{c}{$\mathrm{O}-\mathrm{CH}_{2}-\mathrm{O}$} & $\mathrm{OH}$ & $\mathrm{H}$ & $\mathrm{H}$ \\
$\mathbf{5 b}$ & $\mathrm{H}$ & $\mathrm{O}-\mathrm{Glu}$ & $\mathrm{OMe}$ & $\mathrm{H}$ & $\mathrm{H}$ & $\mathrm{H}$ \\
$\mathbf{5 c}$ & $\mathrm{H}$ & $\mathrm{OMe}$ & $\mathrm{OH}$ & $\mathrm{OMe}$ & $\mathrm{H}$ & $\mathrm{H}$ \\
$\mathbf{5 d}$ & $\mathrm{H}$ & $\mathrm{OMe}$ & $\mathrm{OH}$ & $\mathrm{OMe}$ & $\mathrm{OMe}$ & $\mathrm{H}$ \\
$\mathbf{5 e}$ & $\mathrm{OH}$ & $\mathrm{OMe}$ & $\mathrm{OH}$ & $\mathrm{OMe}$ & $\mathrm{OMe}$ & $\mathrm{OH}$ \\
$\mathbf{5 f}$ & $\mathrm{H}$ & $\mathrm{OMe}$ & $\mathrm{OMe}$ & $\mathrm{H}$ & $\mathrm{OMe}$ & $\mathrm{OMe}$ \\
$\mathbf{5 g}$ & $\mathrm{H}$ & $\mathrm{OMe}$ & $\mathrm{OMe}$ & $\mathrm{H}$ & $\mathrm{OMe}$ & $\mathrm{OH}$
\end{tabular}

5

PROMORPHINANE 6<smiles>[R4]C1=CC2CC([R])C([R])=CC34CCCN(CC3)C4C2=CC1=O</smiles>

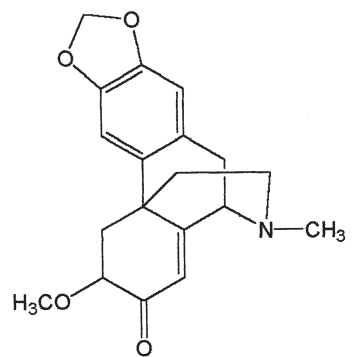

$$
\begin{array}{lcccc} 
& \mathrm{R}_{1} & \mathrm{R}_{2} & \mathrm{R}_{3} & \mathrm{R} \\
\mathbf{6 a} & \mathrm{H} & \multicolumn{2}{c}{\mathrm{O}-\mathrm{CH}_{2}-\mathrm{O}} & \mathrm{OMe} \\
\text { 6d } & \mathrm{OH} & \mathrm{OMe} & \mathrm{H} & \mathrm{OMe}
\end{array}
$$

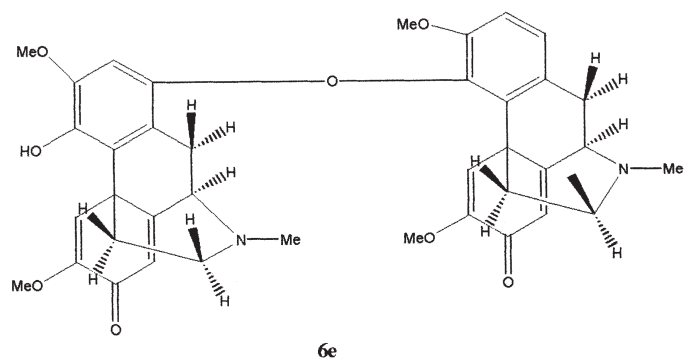

(C) 2002 IUPAC, Pure and Applied Chemistry 74, 557-574 


\section{MORPHINANE 7}
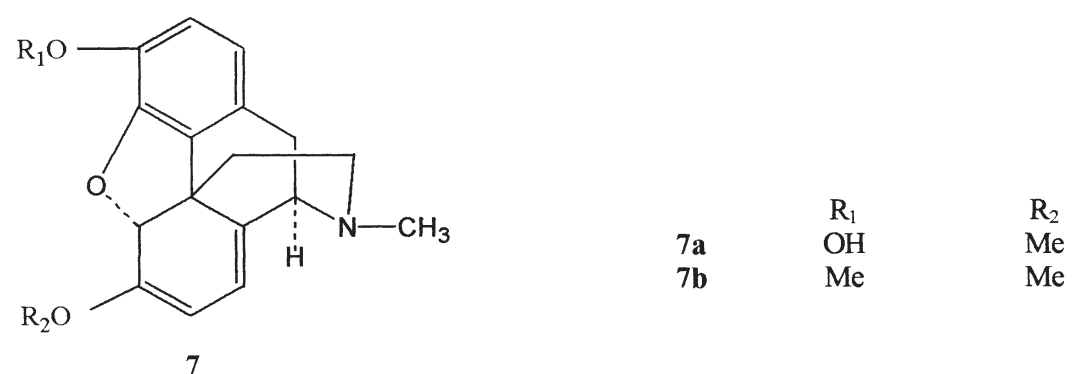

\section{SECOBERBINE 8}<smiles>[R]c1c(C([2H])([2H])C2c3c(cc4c(c3OC)OCO4)CCN2C)ccc(OC)c1OC</smiles>

8

$\begin{array}{lcc} & \mathrm{R}_{1} & \mathrm{R}_{2} \\ \mathbf{8 a} & \mathrm{CH}_{2} \mathrm{OH} & \mathrm{H} \\ \mathbf{8 b} & \mathrm{CHO} & \mathrm{H} \\ \mathbf{8 c} & \mathrm{COOH} & \mathrm{H} \\ \mathbf{8 d} & \mathrm{CH}_{2} \mathrm{OH} & \mathrm{OCOCH}_{3} \\ \mathbf{8 e} & \mathrm{CHO} & \mathrm{OCOCH}_{3} \\ \mathbf{8 f} & \mathrm{COOH} & \mathrm{OCOCH}_{3} \\ \mathbf{8 g} & \mathrm{CH}_{2} \mathrm{OH} & \mathrm{OH}\end{array}$

PROTOBERBERINE 9<smiles>COc1ccc2cc3[n+](cc2c1O)CCc1cc2c(cc1-3)OCO2</smiles><smiles>[R]c1c(OC)cc2c(c1[R])CC1c3c(cc4c(c3OC)OCO4)CCN1C2</smiles>

$\mathrm{R}$

9a $\mathrm{OCH}_{3}$

9b $\mathrm{H}$<smiles>COc1ccc2c(c1OC)CN1CCc3cc4c(c(O)c3C1C2O)OCO4</smiles>

9c<smiles>[R]C(O)COCC([R])O</smiles> 


\section{PROTOPINE 10}<smiles>[R3]c1ccc(CC(=O)c2c(CCN(C)C)cc3c(c2[R])OCO3)c([R2])c1C</smiles>

$\begin{array}{lll}\mathrm{R}_{1} & \mathrm{R}_{2} & \mathrm{R}_{3}\end{array}$

10a $\mathrm{H} \quad \mathrm{O}-\mathrm{CH}_{2}-\mathrm{O}$

10b $\mathrm{H}$ OMe $\mathrm{OMe}$

10c $\mathrm{OMe} \mathrm{OMe} \mathrm{OMe}$

\section{PHTHALIDEISOQUINOLINE 11}<smiles>COC1=C(OC)C2C(C=C1)C1OC2C2c3c(cc4c(c3OC)OCO4)CCN(C)C12O</smiles>

$11 \mathrm{a}$<smiles>[R20]c1c2c(cc3c1N(C)CC3)C1C(=O)OC2OC2C(OC)=C(OC)C=CC21</smiles>

$\mathrm{R}$

11b $\mathrm{OMe}$

11c $\mathrm{OH}$<smiles>COC1=C2C(OC)=C(OC)C3C(=O)OC(C2C=C1)C1CC[N+]3(C)CCc2cc3c(c(OC)c21)OCO3</smiles>

11 


\section{SPIROBENZYLISOQUINOLINE 12}<smiles>[R]c1cc2c(cc1[R])C1(Cc3ccc4c(c3C1([R3])[2H])OCO4)N(C)CC2</smiles>

$\begin{array}{llll}R_{1} & R_{2} & R_{3} & R_{4}\end{array}$

12a O- $\mathrm{CH}_{2} \mathrm{O}$-O-

12b OH OMe H OCOMe

RHOEADINES 13

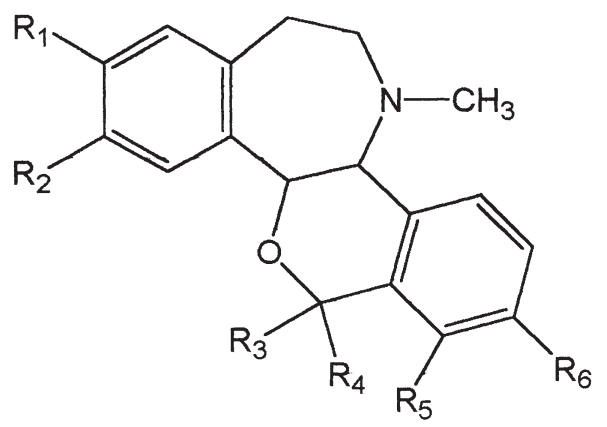

13

13a

$13 \mathrm{~b}$

$13 \mathrm{c}$

13d

$13 \mathrm{e}$

$13 f$

$\mathrm{R}_{1} \mathrm{O}-\mathrm{CH}_{2} \mathrm{O}{ }_{\mathrm{OMe}}^{\mathrm{R}_{2}}$
$\mathrm{OMe}$
$\mathrm{OMe} \mathrm{CH}_{2} \mathrm{O}$
$\mathrm{OMe}$
$\mathrm{OMe}$
$\mathrm{OM} \mathrm{CH}_{2} \mathrm{O}$

$\mathrm{R}_{3}$

$\mathrm{H}$

$\mathrm{H}$

$\mathrm{H}$

$\mathrm{H}$

$\mathrm{OH}$

$\mathrm{H}$
$\mathrm{R}_{5}$

O- $\mathrm{CH}_{2} \mathrm{O}$

$\mathrm{O}-\mathrm{CH}_{2-} \mathrm{O}$

O- $\mathrm{CH}_{2} \mathrm{O}$

O- $\mathrm{CH}_{2} \mathrm{O}$

$\mathrm{O}-\mathrm{CH}_{2} \mathrm{O}$

$\mathrm{OMe} \quad \mathrm{OMe}$ 